
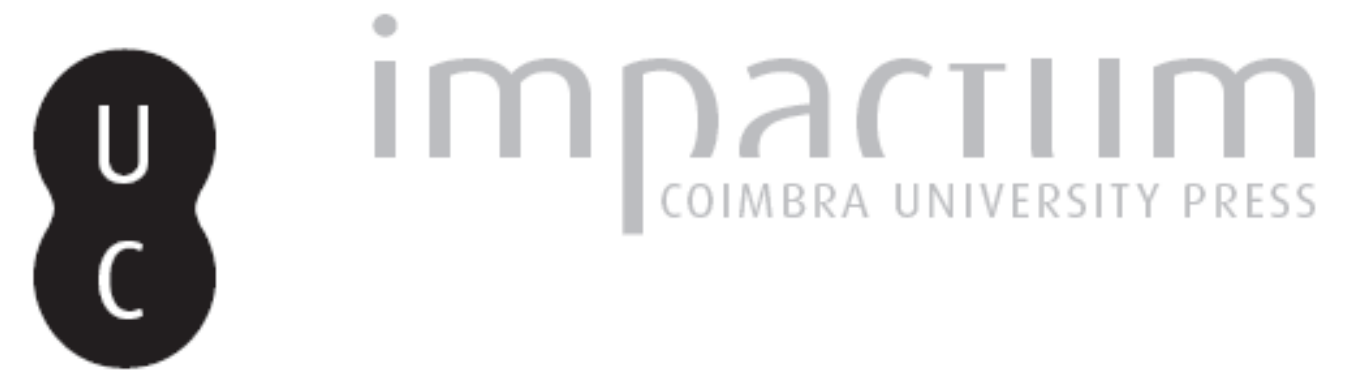

Perché è un buonviatico: la poesia e i poeti nei racconti di Antonio Tabucchi

Autor(es): Bettini, Clelia

Publicado por: Imprensa da Universidade de Coimbra

URL persistente:

URI:http://hdl.handle.net/10316.2/42436

DOI: $\quad$ DOI:https://doi.org/10.14195/0870-8584_8_11

Accessed : $\quad$ 26-Apr-2023 13:38:39

A navegação consulta e descarregamento dos títulos inseridos nas Bibliotecas Digitais UC Digitalis, UC Pombalina e UC Impactum, pressupõem a aceitação plena e sem reservas dos Termos e Condições de Uso destas Bibliotecas Digitais, disponíveis em https://digitalis.uc.pt/pt-pt/termos.

Conforme exposto nos referidos Termos e Condições de Uso, o descarregamento de títulos de acesso restrito requer uma licença válida de autorização devendo o utilizador aceder ao(s) documento(s) a partir de um endereço de IP da instituição detentora da supramencionada licença.

Ao utilizador é apenas permitido o descarregamento para uso pessoal, pelo que o emprego do(s) título(s) descarregado(s) para outro fim, designadamente comercial, carece de autorização do respetivo autor ou editor da obra.

Na medida em que todas as obras da UC Digitalis se encontram protegidas pelo Código do Direito de Autor e Direitos Conexos e demais legislação aplicável, toda a cópia, parcial ou total, deste documento, nos casos em que é legalmente admitida, deverá conter ou fazer-se acompanhar por este aviso. 


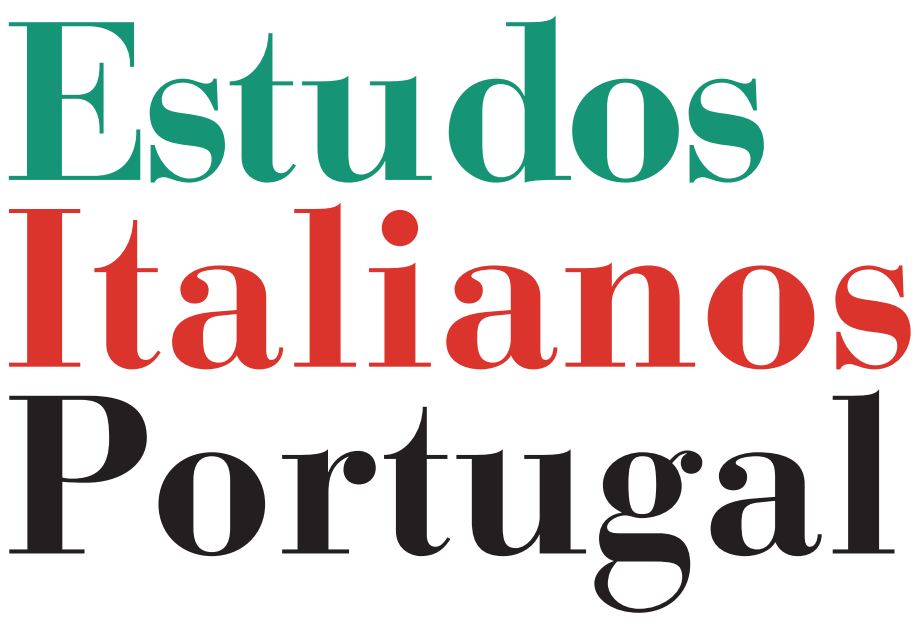

Instituto

Italiano

de Cultura

de Lisboa

Nova Série

$\mathbf{N}^{\circ} \mathbf{8}$ 


\title{
PERCHÉ È UN BUONVIATICO. LA POESIA E I POETI NEI RACCONTI DI ANTONIO TABUCCHI
}

\section{Clelia Bettini*}

\begin{abstract}
Sono un poeta, [...] nella solitudine della mia stanza, apro le botole dell'anima, guardo nel buio dei sotterranei, ci sono topi, ruscelli di diamante, bellezze, miasmi e rancori: lo faccio per me, lo faccio per voi, perché ci vuole qualcuno che guardi, e questi sono i poeti, che cercano le stelle in fondo ai pozzi.
\end{abstract}

Antonio Tabucchi ${ }^{1}$

Antonio TABucchi non ha mai pubblicato poesie. Non è una cosa strana, gran parte dei narratori del Novecento non l'ha fatto. Il che non vuol dire che non abbiano scritto poesia, tantomeno che non ne abbiano letta. Significa solo che hanno trovato nella prosa la propria dimensione artistica, il luogo dove meglio riuscivano a esprimere quello che avevano urgenza di dire. La letteratura è una cosa sola, almeno così ci racconta la sua storia. E nasce come poesia, come narrazione in verso del mondo. I greci narrano la propria storia in esametri, secondo un ritmo ben preciso che scandisce lo scorrere del tempo umano e mettono in scena le loro tragedie e le loro commedie in trimetri giambici. La poesia antica è così antropica che la sua unità metrica viene chiamata "piede", come se non di sillabe si trattasse, ma di minuscoli arti che scandiscono il percorso di un essere umano in carne e ossa. A volte

* Borsista di post Dottorato della FCT.

${ }^{1}$ Il signor Pirandello è desiderato al telefono, Dialoghi mancati, Milano, Feltrinelli, (1988) 1996, pp. 19-20. 
può persino essere un "piede zoppo", come nel caso di quel trimetro giambico "scazonte" che ha sempre fatto sorridere gli studenti dei licei. Sin dai suoi inizi la letteratura — almeno quella occidentale — si muove col passo malfermo della poesia. Con essa si detiene sull'orlo segreto delle cose, sospesa nel vuoto, oppure sprofonda negli abissi più oscuri dell'umano, mano nella mano con quest'arte antica della parola esatta e misteriosa. Si prenda ad esempio il caso di Victor Hugo e, più concretamente di Notre Dame de Paris. In un bellissimo saggio sulla "cattedrale" hugoliana, Donata Feroldi ha svelato tutta la potenza di quello che lo stesso Hugo definiva "romanzo-poema" ${ }^{2}$. Le riflessioni di Feroldi sul ritmo della prosa hugoliana - che la studiosa e traduttrice ha potuto comprendere a pieno solo cimentandosi a lungo con la traduzione del testo in lingua italiana - non lasciano spazio al benché minimo dubbio riguardo alla poeticità di quella che è a tutti gli effetti, un'opera chiave per la narrativa occidentale contemporanea. E la cosa più affascinante è avere la certezza di aver letto poesia senza saperlo, divorando una dopo l'altra le pagine di Notre Dame de Paris. La poesia è così, è un demone che ci abita e da cui ci lasciamo abitare. Spesso arriva sotto forma di malinconia, di quella "tristezza che ai naviganti intenerisce il cuore". A volte è gioiosa, cristallina, ci porta in dono tutti i colori e le luci della vita. E da assuefazione, per fortuna.

In questo articolo vorremmo riflettere sulla presenza della poesia e dei poeti all'interno della narrativa tabucchiana. Per ovvie ragioni di spazio, abbiamo deciso di limitare il corpus testuale di cui occuparci alle prime raccolte di racconti pubblicate da Antonio Tabucchi. L'idea ci è stata suggerita dal primo libro uscito postumo di Tabucchi, pubblicato nel 2013 a cura di Anna Dolfi, Di tutto resta un poco ${ }^{3}$. Il titolo è

\footnotetext{
${ }^{2}$ La chiave della porta rossa. Leggere Victor Hugo, Ancona, Pequod, 2008.

${ }^{3}$ Milano, Feltrinelli, 2013.
} 
stato scelto dall'autore poco prima della sua scomparsa ed è la traduzione italiana di un verso del poeta brasiliano Carlos Drummond de Andrade. Si tratta di una raccolta di saggi su diversi argomenti, fra i quali anche la poesia e i poeti che hanno avuto, per diverse ragioni, un ruolo importante nella vita dello scrittore. Volendo parlare di Tabucchi e della poesia, è immediato pensare a Fernando Pessoa, poeta con cui lo scrittore ha dialogato, su vari livelli, in modo costante per tutto l'arco della sua vita. Tuttavia, parleremo solo tangenzialmente del più importante poeta portoghese del Novecento, perché meriterebbe uno studio a parte. Ma torniamo a Drummond e, per subito venir meno ai nostri propositi, a Pessoa.

Di tutto resta un poco raccoglie una serie di scritti dedicati alla letteratura e al cinema. Fra questi ce n’è uno proprio su Drummond, pubblicato per la prima volta quasi trent'anni fa come introduzione a Sentimento del mondo (1987), antologia in lingua italiana che raccoglie alcune delle più significative poesie di Drummond, tradotte da Tabucchi stesso ${ }^{4}$. Si tratta di un testo breve ma rivelatore del cuore poetico tabucchiano:

con Pessoa del resto Drummond presenta più che una affinità $[. .$.$] an-$ che un'affinità generazionale: entrambi appartengono alla generazione che ha fatto (o fiancheggiato) le avanguardie storiche; che ha costruito la poesia moderna eppure, allo stesso tempo, ha tenuto in sospetto, con lo sguardo dell'ironia, il culto del moderno. ${ }^{5}$

Accostare Drummond a Pessoa significa in primo luogo dichiararne il peso specifico. Ai lettori che già conoscono il poeta portoghese attraverso i due volumi de Una sola moltitudine (1979) e Il libro dell'inquietudine di Bernardo Soares (1986), Tabucchi suggerisce una pista da percorrere, un

${ }^{4}$ Cfr. Carlos Drummond de Andrade, Sentimento del mondo. Trentasette poesie scelte e tradotte da Antonio Tabucchi, Torino, Einaudi, 1987, pp. V-VI.

${ }^{5}$ Di tutto resta un poco, p. 89. 
movimento del pensiero importante da compiere. Potremmo dire che sono entrambi poeti che hanno fatto le avanguardie come Ungaretti ha fatto la guerra, suo malgrado, e, come Ungaretti, da quell'esperienza per certi versi violenta hanno tratto materia poetica originale, silenziosamente rivoluzionaria. Sono poeti provvisti d'ironia — una delle qualità più importanti per uno scrittore, secondo Tabucchi — caratteristica che ha permesso loro di continuare a osservare l'umano senza lasciarsi soffocare dai rombi del modernismo.

Dopo aver chiarito dove non si è collocato storicamente Drummond, Tabucchi indica al lettore in quale costellazione poetica lo si debba inserire. In particolare quando spiega come Drummond sia un poeta dell'inadeguatezza che si afferma come "antieroe" che racconta il quotidiano. Il che equivale a dire che non appartiene a quella schiera di poeti che si muovono fra "bossi ligustri o acanti", ma a quella di coloro che traggono la propria ispirazione dalle cose semplici, dal "niente":

Un niente testardo, quello di Drummond, che non muore, che resiste, che circola nei canali più ingrati della vita, attraverso giuncaie e pantani, risalendoli come l'anguilla di Montale, per deporre il suo poco. Perché "di tutto resta un poco" (Resíduo), ed è con questo poco, che poi è il nostro tutto, che dobbiamo fare i conti. ${ }^{6}$

Ed ecco che con questa perla residuale, Tabucchi indica una pista poetica da seguire. Ci invita a imboccare una di quelle "strade che riescono agli erbosi/fossi dove in pozzanghere/mezzo seccate agguantano $\mathrm{i}$ ragazzi/qualche sparuta anguilla" 7 . Ci vuole portare là, in quegli orti, tra gli alberi dei limoni. Pessoa come Drummond. Drummond come Montale. E come Tabucchi, perché vista con il senno del poi, la scelta del titolo di quest'ultimo suo libro è un segno del fatto

${ }^{6}$ Ib., p. 91.

${ }^{7}$ Cfr. Eugenio Montale, Ossi di seppia, Torino, P. Gobetti, 1925 (1. ${ }^{\mathrm{a}}$ ed.). 
che anch'egli si trovava a suo agio negli stessi luoghi letterari. È diventata, implicitamente, una vera e propria dichiarazione di poetica.

Il rancore e le nuvole (Piccoli equivoci senza importanza) è un racconto su certi personaggi che circolano in quegli strani universi paralleli che sono le Facoltà di Lettere. Tabucchi vi ha insegnato a lungo, seppure sempre con lo sguardo dell'outsider che gli veniva dall'essere soprattutto uno scrittore. $\grave{E}$ un ritratto atroce, difficile da leggere per chi in quelle università ha studiato e lavorato, ma anche molto lucido $\mathrm{e}$ veritiero. Non è l'unico racconto dedicato a questo mondo che di letteratura si occupa davvero, un'istituzione in cui Tabucchi ha sempre creduto fermamente. Evidentemente l'argomento gli stava a cuore, per usare un eufemismo ${ }^{8}$. Vi si descrive la parabola "ascendente" di uno studente di filologia che con spregiudicata meditazione riesce a diventare un professore affermato. La poesia è lo strumento grazie al quale il protagonista mette in atto la conquista di un'identità che è certo più sociale che intima. Essa diviene simbolo della sua determinazione nel riuscire a diventare ciò che vuole e si condensa nel ritratto fisico di Antonio Machado che il protagonista porta con sé, per poi infine appenderlo "in un luogo decoroso, vicino a libri degni di lui" nel suo studio in un bell'appartamento in centro9. La fotografia di Machado è a sua volta simbolo della celebre poesia Retrato del poeta spagnolo, citata esplicitamente nel racconto, perché il protagonista in essa ha deciso di identificarsi. Nonostante la manifesta malevolenza che guida le sue azioni (l'abbandono di una famiglia troppo triste e dimessa, la vendetta sotto forma di disprezzo del Nostalgico, il vecchio professore grazie al quale è riuscito a fare carriera), egli si riconosce nei versi

\footnotetext{
${ }^{8}$ A questo proposito si veda P. Abbrugiati, “Buoni come sono. Tabucchi et les brebis noires de l'académie”, Italies, 11, 2007 (Aix-en-Provence: Centre Aixois d'Études Romanes, CAER), pp. 165-192.

${ }^{9}$ Piccoli equivoci senza importanza, Milano, Feltrinelli, 1999 (1. a ed. 1985), p. 97.
} 
di Machado che descrivono, indubbiamente, il suo opposto umano: "hay en mis venas gotas de sangre jacobina; Pero mi verso brota de manantial sereno; Y más que un hombre al uso que sabe su doctrina; Soy, en el buen sentido de la palabra, bueno" 10 . La carica ironica che scaturisce dalla citazione di Machado, tremula oltre le lacrime che velano lo sguardo emozionato di quel piccolo rapace della cultura, è fortissima. La poesia diviene una specie di cuore rivelatore e il suo battito pulsa riflesso in uno specchio, deve essere letto al rovescio.

Ora, ciò che dal nostro punto di vista risulta forse più interessante è che l'intero racconto si sviluppa entro una cornice drummondiana. Si tratta della poesia Conclusão, che riporto per intero:

Os impactos de amor não são poesia (tentaram ser: aspiração noturna).

A memória infantil e o outono pobre vazam no verso de nossa urna diurna.

Que é poesia, o belo? Não é poesia, e o que não é poesia não tem fala.

Nem o mistério em si nem velhos nomes poesia são: coxa, fúria, cabala.

Então, desanimamos. Adeus, tudo!

A mala pronta, o corpo desprendido, resta a alegria de estar só, e mudo.

De que se formam nossos poemas? Onde?

Que sonho envenenado lhes responde,

se o poeta é um ressentido, e o mais são nuvens? ${ }^{31}$

Come si vede, il titolo del racconto di Tabucchi è una rielaborazione dei versi finali della Conclusão drummundiana, che è in sé una riflessione sul senso profondo dell'essere poeta. Ed è sull'autore di questi versi, un poeta definito "asciutto e realistico", che il protagonista - all'inizio del racconto - si appresta a scrivere l'ennesima comunicazione per l'ennesimo congresso.

${ }^{10} I b .$, p. 88.

${ }^{11}$ Carlos Drummond de Andrade, Fazendeiro do ar, Rio de Janeiro, José Olympio, 1954. 
Come per incanto, dall'analisi "ritmica" della poesia di Drummond nasce la narrazione della vita di questo losco figuro.

È importante ricordare che Tabucchi non rivela il nome del poeta chiamato in causa e non ne parla "a modo suo". Si limita a far parlare di lui il suo personaggio. Così si scopre che, tutto sommato, è un poeta che non gli dispiace, "anche se forse appannato da una venatura metafisica che trovava superflua" ${ }^{\prime 2}$. Risulta evidente che il filologo professore, in realtà, disdegna l'autore su cui ha deciso di scrivere. Ritiene che abbia composto quei versi "senza capirne il significato" e arriva a esautorarlo, sostituendosi a lui in virtù di quel risentimento che ha dominato la sua esistenza. E l'analisi sprezzante della poesia di Drummond - riassunta nel paradigmatico titolo della comunicazione (Il rancore e le nuvole. Per una lettura ritmica di una poesia del Novecento) - se opportunatamente riflessa in uno specchio, diventa la chiave per comprendere l'intero racconto.

Nel caso de Il rancore e le nuvole la poesia di Drummond ha una duplice funzione: da un lato serve da innesco alla narrazione, dall'altro la illumina di senso. È una modalità che ritroviamo anche in altri racconti di Tabucchi. Ne è un esempio il breve post-scriptum posto in calce ai racconti di Donna di Porto Pim, il cui sottotitolo recita Una balena vede gli uomini ${ }^{13}$. L'innesco drummondiano è rivelato dall'autore nel "Prologo" del libro:

Infine lo scritto intitolato Una balena vede gli uomini, al di là di un mio vecchio vizio di spiare le cose dall'altra parte, si ispira senza dissimulazione a una poesia di Carlos Drummond de Andrade, che prima e meglio di me ha saputo vedere gli uomini attraverso gli occhi penosi di un lento animale. E a Drummond quel testo è umilmente dedicato, anche in ricordo di un pomeriggio a Ipanema in cui, in casa di Plínio Doyle, egli mi parlò della sua infanzia e della cometa di Halley. ${ }^{14}$

\footnotetext{
${ }^{12}$ Piccoli equivoci senza importanza, p. 98.

${ }^{13}$ Donna di Porto Pim, Palermo, Sellerio, 1996 (1. a ed. 1983), pp. 89-90.

${ }^{14}$ Ib., p. 10.
} 
La poesia di Drummond a cui Tabucchi si riferisce è $U m$ boi vê os homens, quasi un poème en prose, dove il poeta immagina come un bue veda gli umani ${ }^{15}$. Così come accadeva per l'introduzione a Sentimento del mondo, anche questa breve nota di Tabucchi ha la funzione di una dichiarazione di poetica. Come Drummund, afferma di avere il vizio di "spiare le cose dall'altra parte", per raccontare il rovescio (o i rovesci) di una "visione della realtà imposta dal pensiero dominante"16. E questo "vizio", come è noto, costituisce uno dei cardini sui cui ruota la narrativa tabucchiana. Appare insomma evidente, a questo punto, che Drummond sia stato d'importanza fondamentale per la costruzione di una personalissima poetica letteraria. Ecco perché non stupisce rincontrarlo anche ne L'angelo nero, raccolta di racconti dove la poesia scorre in impetuosi fiumi sotterranei.

L'angelo nero rappresenta un punto di svolta all'interno della narrativa di Tabucchi, come spiega egli stesso in una lunga intervista rilasciata al suo traduttore spagnolo, Carlos Gumpert ${ }^{17}$. L'angelo nero nasce dalla necessità di confrontarsi con la realtà del male che, secondo Tabucchi, può essere narrata solo "a partir de cierto momento de la vida, porque cuando se es joven ésta se nos presenta de modo más solar, más luminoso. En cambio, sus aspectos más oscuros y sórdidos se captan mejor con el paso de los años" ${ }^{\prime 1}$. Tutti i racconti hanno in comune l'indagine del lato oscuro della vita, sono essi stessi angeli neri, dal "pelame raso, che punge"19. Ogni possibile dubbio sull'origine montaliana del titolo della raccolta è fugato da Tabucchi stesso: "Il titolo di questo libro appartiene a Eugenio Mon-

\footnotetext{
${ }^{15}$ Cfr. Claro enigma, Rio de Janeiro, José Olympio, 1951.

${ }^{16}$ Cfr. Di tutto resta un poco, p. 12.

${ }_{17}$ Conversaciones con Antonio Tabucchi, Barcelona, Anagrama, 1995. Su L'angelo nero (El ángel negro) si vedano in particolare le pp. 177-185.

${ }^{18} \mathrm{Ib}$., p. 178.

${ }^{19}$ L'angelo nero, Milano, Feltrinelli, 1999 (1. ${ }^{\text {ed }}$ ed 1991), p. 9.
} 
tale, che prima di me si è imbattuto in un angelo dalle ali nere"20. In primo luogo, dunque, lo scrittore stabilisce un rispettoso diritto di precedenza, grazie al quale rende elegantemente omaggio al poeta scomparso. In secondo luogo, però, Tabucchi ricorre a Montale per parlare di sé, stabilisce una corrispondenza con il poeta ligure, come già era successo nel momento in cui si era trovato a parlare di Drummond de Andrade.

E il poeta brasiliano torna a essere citato in Notte, mare $o$ distanza, il cui titolo traduce un verso della poesia drummondiana Noturno à janela do apartamento: "suicídio, riqueza, ciência.../A alma severa se interroga/e logo se cala. E não sabe/se é noite, mar ou distância" ${ }^{\prime 2}$. È il racconto del ricordo di una notte atroce nella vita di quattro giovani amici, costretti a confrontarsi con l'orrore e l'arroganza della dittatura salazarista. Siamo nel 1969 e ancora una volta è Drummond a innescare la narrazione, perché i ragazzi hanno trascorso una lunga serata a parlare di poesia a casa di un amico poeta, più vecchio di loro, di nome Tadeus ${ }^{22}$. La poesia fa loro da scorta, quando si trovano ad affrontare la difficoltà del vivere, perché, come dice Tadeus, la poesia "è un buon viatico".

Fra i poeti che hanno letto e discusso quella notte, c'è anche Drummond e uno dei personaggi recita, storpiato, il verso drummondiano che dà il titolo al racconto ${ }^{23}$. Lo sgomento del Noturno drummondiano è lo stesso che sentono quei giovani prigionieri della lunga notte del salazarismo, almeno nel ricordo che di quel tempo ha il narratore.

${ }^{20} \mathrm{Ib}$., p. 10.

${ }^{21}$ Cfr. Sentimento do mundo. Poesia, Rio de Janeiro, José Olympio, 1948.

22 Tadeus è un personaggio che vaga come un fantasma inquieto attraverso la narrativa tabucchiana. Si veda ancora Voci portate da qualcosa, impossibile dire cosa, sempre in L'angelo nero, ma anche Antonio Tabucchi, Requiem. Un'allucinazione, Milano, Feltrinelli, 1991, pubblicato a breve distanza dalla raccolta di racconti citata.

${ }^{23}$ L'angelo nero, p. 33. 
No, lo sapevano tutti che restavano per altro [a casa di Tadeus], proprio perché fuori era notte, mare o distanza e la frase di Tiago aveva manifestato un sentimento che era di tutti e che nessuno aveva il coraggio di rendere esplicito: un disagio come una tenue malattia; non paura; piuttosto un misto di insicurezza e di struggimento, come sentirsi profughi in una città che era la loro e avere nostalgia della loro vera città, che era quella stessa, ma in un altro momento che non fosse quella sera ostile, quella notte, con le sue onde malefiche che vibravano pronte a scatenarsi. ${ }^{24}$

Anche in questo caso, come già era accaduto in altri racconti, la poesia di Drummond mette in moto la narrazione, ma allo stesso tempo permette di comprendere il senso profondo del testo tabucchiano, come rivela in modo chiaro il dialogo che lo scrittore instaura con la lirica Noturno à janela de um apartamento.

Oltre a Drummond, un altro poeta compare in questo racconto. E facile infatti riconoscere in Tadeus il poeta portoghese Alexandre O'Neill ${ }^{25}$. Il più vecchio dei giovani protagonisti, proprio quella sera ha ritirato dalla tipografia una raccolta di sue poesie che sta per uscire in libreria. Nel 1969 O'Neill pubblica la raccolta De ombro na ombreira e non è un caso che ritroviamo Tadeus, per ben due volte, "con la spalla appoggiata allo stipite" 26 . E la magica, sinistra apparizione di una cernia, che agonizzante partecipa agli eventi è un altro

\footnotetext{
${ }^{24} I b .$, p. 33.
}

${ }^{25}$ Secondo Tabucchi, Alexandre O’Neill è “uno de los mayores autores portugueses de la pos-guerra, por desgracia poco conocido fuera de su país. Es un poeta de una gran carga subversiva anárquica y de un notable sentimiento de conmoción y de pena hacia el mundo, con una gran capacidad de autoironía y de escarnio respecto a las ideas tópicas y la metalidad corriente, que ha escrito una poesía de altísima calidad" (Carlos Gumpert, Conversaciones con Antonio Tabucchi, p. 54). Anche di Alexandre O’Neill, come di Carlos Drummond de Andrade, Tabucchi ha pubblicato un'antologia in traduzione italiana. Cfr. Alexandre O'Neill, Made in Portugal, a cura di Antonio Tabucchi, Milano, Guanda, 1978.

${ }^{26}$ L'angelo nero, pp. 43 e 48. 
chiaro richiamo alla poesia di O'Neill ${ }^{27}$. Tuttavia, è bene ricordarlo, Tadeus è un personaggio letterario e la poesia e la vita di O'Neill divengono anch'esse personaggi di finzione all'interno della narrativa, a prescindere da qualunque dato di realtà che ne risieda alla base. Ed è da questo presupposto che deve muoversi l'analisi di un altro racconto "poetico" incluso in L'angelo nero.

Si tratta de La trota che guizza fra le pietre mi ricorda la tua vita, racconto che narra la storia di un vecchio poeta, ormai giunto alla fine dei suoi giorni. È un poeta ironico, che ama prendersi gioco degli altri ma soprattutto di se stesso. Riceve a pranzo una giovane poetessa e critica letteraria dai capelli biondi, di cui gli importa poco o nulla. E dialoga in sogno con la propria vita passata.

Il titolo del racconto non è un verso di Montale. Potremmo però dire che è un verso montaliano. C'è un animale che guizza, come l'anguilla dell'omonima lirica già citata a proposito di Drummond ${ }^{28}$. Ci sono le pietre che evocano le pietraie d'un greto dove scorre l'acqua limpida di Ripenso il tuo sorriso $^{29}$. C'è il ritmo sospeso della poesia di Montale, delle parole e delle immagini che si susseguono senza obbedire alla metrica regolare dei "poeti laureati".

In particolare, però, il riferimento alla trota rinvia tematicamente alla poesia La trota nera, una delle liriche di Montale comprese nel Diario postumo ${ }^{30}$, ultima polemica raccolta del poeta ligure, su cui molti critici hanno avanzato non pochi dubbi. La storia editoriale della postuma raccolta montaliana è nota. Il poeta aveva lasciato le 66 liriche come "testamento poetico" all'amica poetessa (e ultima musa) Annalisa Cima, con l'indicazione di pubblicarne sei ogni anno, a partire dalla

${ }^{27}$ Si tratta della poesia Sigamos o cherne! (após ver o filme), compresa nella raccolta No reino da Dinamarca, Lisboa, Guimaräes Editores, 1958 (1. ${ }^{\mathrm{a}} \mathrm{ed}$.).

${ }^{28}$ Cfr. Eugenio Montale, La bufera e altro, Venezia, Neri Pozza, 1956 (1. a ed.).

${ }^{29}$ Cfr. id., Ossi di seppia.

${ }^{30}$ Cfr. id., Diario postumo, Milano, Mondadori, 1996. 
sua morte. Ed ecco un'altra coincidenza montaliana, perché il vecchio poeta del racconto di Tabucchi regala anch'egli un madrigale alla giovane poetessa bionda che lo va a trovare.

Il protagonista del racconto ricorda i tempi in cui ancora la "trota guizzava fra le pietre", la stessa trota salmonata che ora giace adagiata su un piatto da portata, insieme a qualche verdura bollita sulla sua tavola da pranzo. Le donne che quel tempo hanno abitato vengono a fargli visita, tabucchianamente, fra il sonno e la veglia. Hanno nomi d'altri tempi, Lydia e Lucrezia e lo scherniscono con dolcezza, forti di un'intelligenza di cui difetta la poetessa-ragazza che lo attende in sala da pranzo.

Indubbiamente, in questo racconto, Tabucchi immagina un frammento della vecchiaia di un poeta che ricorda moltissimo Montale, lo inventa e non ha alcuna pretesa di verità. A questo proposito, scrive:

"La scienza è rozza, la vita è sottile, ed è per correggere questa distanza che la letteratura ci interessa."

La vita è sottile, è vero, ma aggiungerei anche che è insufficiente: "La letteratura, come tutta l'arte, è la dimostrazione che la vita non basta"(Fernando Pessoa). La letteratura offre la possibilità di un di più rispetto a ciò che la natura concede. ${ }^{31}$

Dunque quel vecchio poeta non è Montale. Se anche forse lo è stato in parte nella fase progettuale (o dell' ispirazione) del racconto, ha smesso di esserlo nel momento in cui è divenuto personaggio. Come accade a qualunque altro personaggio letterario. E un tema su cui Tabucchi ha lasciato indicazioni precise, soprattutto in quel libro di profonda autoanalisi che è Autobiografie altrui (poetiche a posteriori):

[...] in queste quintessenze che sono i personaggi, è inutile cercare ciò che appartiene all'ufficio dell'anagrafe. Senza scomodare le più recen-

${ }^{31}$ Di tutto resta un poco, p. 15, cit. di Roland Barthes. 
ti teorie della letteratura, mi limiterei a un'impareggiabile riflessione sull'argomento che Carlo Emilio Gadda ebbe a fare assai prima che le discipline narratologiche elaborassero la formulazione della cosiddetta "autonomia del personaggio": “... Io vagheggio con la fantasia di una certa signora X, un 'mio' personaggio: la vagheggio fino a sognarmela di notte: mi sveglio di soprassalto, mi levo dal letto in stato di trance, siedo al tavolo, scrivo: dopo mesi e mesi riprendo quel foglio, riscrivo, gratto, cancello, riscrivo: ricopio quaranta volte: lo dò all'editore. La signora X è venuta al mondo. Succede che a Brembate o a Garbagnate, c'è davvero una signora tale e quale come la signora X. Si tratta, come ognuno capisce, di un incidente combinatorio: che cade sotto il principio di indeterminazione assoluta o principio di Heisemberg. Come quando due giocatori, giocando ai dadi, gli viene cinque e tre a tutt'e due. Io, nel mio cervello, nella mia psiche ho creato: ho maturato lentamente la signora $\mathrm{X}$ mentre con eguale ponderatezza il Padre Eterno, a Garbagnate, ha maturato per suo conto un'altra signora, che tra tutt'e due, però, si somigliano come due gocce d'acqua". ${ }^{32}$

Nel frammento citato da Tabucchi, Gadda suggerisce un'idea di contemporaneità tra realtà e creazione letteraria, una specie di coincidenza a priori che non può essere spiegata. E inutile farlo. Ecco perché questo vecchio poeta non è Montale. L'idea di un personaggio nasce sempre dal mondo in cui lo scrittore vive, come potrebbe essere altrimenti? Tuttavia l'invenzione letteraria ha una carica demiurgica indipendente, come suggerisce Gadda. E così Tabucchi ha creato un poeta che somiglia moltissimo a Montale, ma che non è lui.

È montaliano, questo sì, tutto l'universo che egli rappresenta. Come quanto dice tra sé e sé "la poesia è l'abbaglio: questo è la poesia"33, scatenando nel lettore tutta una concatenazioni di corrispondenze montaliane legate all'immagine poetica dell'abbaglio/barbaglio:

\footnotetext{
${ }^{32}$ Milano, Feltrinelli, 2003, pp. 99-100. La citazione di Gadda è tratta da Per favore mi lasci nell'ombra. Interviste 1950-1972, a cura di C. Vela, Milano, Adelphi, 1993.

${ }^{33}$ L'angelo nero, p. 102.
} 
$\mathrm{E}$ andando nel sole che abbaglia sentire con triste meraviglia com'è tutta la vita e il suo travaglio in questo seguitare una muraglia che ha in cima cocci aguzzi di bottiglia. ${ }^{34}$

Ci muoviamo in un pulviscolo madreperlaceo che vibra in un barbaglio che invischia gli occhi e un poco ci sfibra ${ }^{35}$

e mi chiesi se questo che mi chiude ogni senso di te, schermo d'immagini ha i segni della morte o dal passato è in esso, ma distorto e fatto labile, un tuo barbaglio. ${ }^{36}$

La poesia e la poetica di Montale scorrono attraverso il racconto, ma non con l'intento di descrivere in modo realistico un frammento di vita dell'uomo Montale. Così come accade, a dispetto del titolo, in Antero de Quental. Una vita. In merito a questa storia, compresa nella già citata raccolta Donna di Porto Pim, Tabucchi afferma che sebbene racconti nei suoi fatti sostanziali la vita del poeta azzorriano, non sarebbe improprio definirla finzione. E aggiunge:

Devo al suggerimento di Octavio Paz che i poeti non abbiano biografia e che la loro opera sia la loro biografia, l'averla raccontata come se si trattasse di una vita immaginaria. Del resto le vite che si persero per via, come quella di Antero, sono quelle che meglio tollerano di essere narrate secondo i canoni dell'ipotetico. ${ }^{37}$

\footnotetext{
${ }^{34}$ Cfr. Meriggiare pallido e assorto, Ossi di seppia.

${ }^{35} \mathrm{Cfr}$. Non rifugiarti nell'ombra, ib.

${ }^{36}$ Cfr. La speranza di pure rivederti, Le occasioni, Torino, Einaudi, 1939 (1. ${ }^{\text {e ed.). }}$

${ }^{37}$ P. 10.
} 
I poeti e la poesia s'iscrivono dunque a pieno titolo entro $\mathrm{i}$ confini dell'ipotetico, del vagheggiare e del sogno, categorie cruciali della poetica tabucchiana. Ecco perché Tabucchi vi trova materia fondamentale per la sua narrativa. Antero de Quental appartiene alla schiera dei poeti notturni, di coloro che trovano nell'oscurità della sera una armonia che consola l'anima dolente. "Espírito que passas, quando o vento/ adormece no mar e surge a lua,/filho esquivo da noite que flutua,/tu só entendes bem o meu tormento", scrive Antero in un sonetto intitolato, appunto, Nocturno ${ }^{38}$. Foscolo, da parte sua, aveva compreso il perché di questa passione di alcuni poeti per la notte. L'illusione di morte che si materializza ogni giorno al calar del sole mette a tacere "quello spirito guerrier ch'entro mi rugge". Lo "spirito" foscoliano è inquieto, come lo è l'"espírito" notturno di Antero de Quental. E d'Inquietudine parla molte volte Tabucchi, a proposito della letteratura che più ama, perché percepisce questo sentimento come cifra del suo secolo. In particolare ne scrive in Controtempo, saggio incluso nella raccolta Di tutto resta un poco. L'Inquietudine è descritta come la malattia novecentesca per eccellenza che, tuttavia si prolunga "aumentando a dismisura fino a oggi". Secondo Tabucchi essa deriva da "un rapporto sfasato con il Tempo quale dimensione in cui non riusciamo più ad abitare, [da] un rapporto guasto con la Storia"39. Lo scrittore parla ovviamente in primis del Desassossego di Soares/Pessoa, citando il mal de viver del suo personaggio che, in qualche misura, diviene figura auerbachiana del male di vivere di Montale. Ecco dunque che ritorna Montale, in uno degli ultimi scritti di Antonio Tabucchi, e con la potenza che gli è consona, in un abbaglio, svela parte dell'arcano. Nella narrazione dell'Inquietudine risiede la potenza della poesia,

${ }^{38}$ Antero de Quental, Sonetos, organização, introdução e notas de Nuno Júdice, Lisboa, INCM, 1994, p. 70.

${ }^{39}$ Di tutto resta un poco, p. 20. 
di quel desassossego che è "un'estraneità nei confronti della realtà circostante, marcata da una sfumatura che l'avvicina al concetto di spleen baudelairano, di pena per le creature del mondo, di turbamento" 40 .

E di spleen è costellato un altro racconto tabucchiano sul quale merita di soffermarsi, tratto ancora da Piccoli equivoci senza importanza: Anywhere out of the world.

Il racconto parte da "un niente, una frase perduta in questo vasto mondo pieno di oggetti e di volti" ${ }^{2}$. Una frase che è il Niente di Drummond, l'Anguilla di Montale. Una frase che mette in comunicazione i vivi con i morti, il Tempo presente con il suo passato e che è il titolo di uno dei petits poèmes en prose de Le spleen de Paris di Baudelaire ${ }^{42}$. Si tratta di un poemetto che parla di Inquietudine, in particolare di quella che si manifesta nel continuo desiderio di déménagement. $\mathrm{E}$ un'antichissima forma di ansia melancolica, che si ritrova già in Orazio, quando afferma "Romae Tibur amem, ventosus Tibure Romam" (Epistulae I 8), ovvero di avere la certezza di stare bene solo dove non si sta, in qualunque luogo, purché fuori da questo mondo.

Il personaggio di Tabucchi si aggira per Lisbona, primo luogo in cui Baudelaire suggerisce di vivere alla sua anima infreddolita, con cui dibatte la questione del dove trasferirsi. Una città calda, così la immagina Baudelaire, fatta di marmo, di luce minerale che si riflette nelle sue acque. La stessa calura che Tabucchi racconterà in Requiem, lo stesso effetto di sospensione che caratterizza la canicola di Montale (penso a Meriggiare pallido e assorto, Non chiederci la parola, ma soprattutto Arsenio). Il personaggio legge il titolo del poemetto baudelairiano su un giornale, una frase perduta che non può essere lì perché appartiene al passato. Ė una frase segreta della

${ }^{40}$ Ib., p. 21.

${ }^{41}$ Piccoli equivoci senza importanza, p. 71.

${ }^{42}$ Per i testi di Baudelaire, citiamo l'edizione di Alfonso Berardinelli, Lo spleen di Parigi, Milano, Garzanti, 1989. 
cui esistenza sono a conoscenza solo lui e una persona che non c'è più. E da questo momento, Tabucchi cita Baudelaire nel corpo del testo, virgolettato e in francese. In primis trascrive parti del poemetto che dà il titolo al racconto, in particolare la descrizione che lì si fa di Lisbona ${ }^{43}$. In seguito, però, si serve di altri frammenti de Le spleen de Paris. La narrazione del ricordo del protagonista è costruita mediante l'innesto di schegge baudelairiane che a loro volta innescano l'immaginazione del personaggio che racconta, come se sognasse la vita di un suo doppio. Troviamo "une chambre qui ressemble à une rêverie", dove un uomo e una donna sono stati insieme, come nell'assenza di Tempo de La chambre double di Baudelaire che il poeta ottiene grazie al laudano ${ }^{44}$. E quell'uomo dice alla sua compagna "laisse-moi respirer longtemps l'odeur de tes cheveux", incipit de Un hémisphère dans une chevelure ${ }^{45}$. E il resto del dialogo fra i due personaggi, in italiano, scorre rapido, senza citazioni, ma nasconde al suo interno l'incipit di un altro poemetto (L'horloge) che recita così: "les Chinois voient l'heure dans l'oeil des chats" ${ }^{\text {". }}$. Ancora la sospensione del Tempo, la sua indefinitezza così ben descritta da Baudelaire, si riflette nella costruzione narrativa tabucchiana, diventando l'insistenza dell'amante che non vuole lasciar andar via l'amata. E la coscienza dell'impossibilità di quell'amore già vissuto diventa necessità di fuga che ritrova in Baudelaire le parole per essere espressa, tradotte e assimilate, divenute carne e vita di chi le ha lette:

gli stessi gusti: case bianche con palme esigue o una vegetazione rada e essenziale, agavi, tamerici, una roccia; le stesse passioni: Chopin o musiche povere, vecchie rumbe, Tiengo el corazón maluco; la stessa

\footnotetext{
43 Piccoli equivoci senza importanza, pp. 75-76.

${ }^{44}$ Lo spleen di Parigi, pp. 16-17.

${ }^{45}$ Piccoli equivoci senza importanza, p. 78; Lo spleen di Parigi, pp. 68-69.

${ }^{46}$ Lo spleen di Parigi, pp. 66-67.
} 
anima: lo spleen de Paris. Via da qui, da questo spleen, cerchiamo una città bianca, fatta di marmo a fior d'acqua, cerchiamola insieme, una città così o un'altra analoga, non importa dove, da qualche parte, fuori dal mondo. ${ }^{47}$

E il racconto di questo rincontrarsi che non è mai avvenuto, dove Baudelaire diviene il terzo elemento partecipe del codice segreto fra i due personaggi, termina con la rielaborazione di un altro frammento de L'horloge:

Ti siedi sulla spalletta del lungofiume, l'acqua è limacciosa e inquieta, sai che è tardi, ma non nel senso dell'orologio, intorno a te l'ora è vasta, solenne, grande come lo spazio: un'ora immobile che non è segnata sul quadrante, e tuttavia leggera come un sospiro, rapida come un colpo d'occhio. ${ }^{48}$

au fond de ses yeux adorables je vois toujours l'heure distinctement, toujours la même, une heure vaste, solennelle, grande comme l'espace, sans divisions de minutes ni de secondes, - une heure immobile qui n'est pas marquée sur les horloges, et cependant légère comme un soupir, rapide comme un coup d'oeil. ${ }^{49}$

L'Éternité che Baudelaire leggeva negli occhi della sua belle Féline diviene in Tabucchi il Tempo sospeso di ciò che è stato ma torna a far vista a chi lo ha vissuto. Il Tempo della ricerca di una fuga dall'Inquietudine che a priori sappiamo non essere possibile, perché "caelum non animum mutant qui trans mare currunt” (Orazio, Epistulae I XI).

Anche in questo caso la poesia ha la doppia valenza di innesco e rivelazione. Ancora di più rispetto agli altri racconti di cui si è parlato, la poesia diventa qui parte integrante della narrazione. Non è più citata sporadicamente, ma scandisce il ritmo del racconto, lo determina in un modo che può essere letto solo come frutto di grande passione poetica.

\footnotetext{
${ }^{47}$ Piccoli equivoci senza importanza, p. 80, corsivo mio.

${ }^{48} \mathrm{Ib}$., p. 81, corsivo mio.

${ }^{49}$ Lo spleen di Paris, p. 66.
} 
È il momento di tirare qualche conclusione, per quanto provvisoria. Da questa parziale ricognizione della narrativa breve di Antonio Tabucchi si evince che la poesia e i poeti hanno giocato un ruolo importante nella genesi e nella costruzione di alcuni racconti. In primo luogo per la consonanza poetica che Tabucchi sentiva con autori come Drummond o Montale, fautori di un claro enigma che racchiude in sé il senso profondo del mondo. In secondo luogo per una particolare caratteristica che accomuna un certo tipo di poeti, come Drummond e Montale, certo, ma anche Antero, Baudelaire, Pessoa, o Rilke, che spesso compare citato all'interno della narrativa tabucchiana, e Leopardi, e Zanzotto, di cui Tabucchi parla ancora in Di tutto resta un poco. Questo breve periplo poetico porta a riflettere, in un'ultima istanza, sull'Inquietudine. Se ne deduce che una volta presa la si sia contratta, e dall'Inquietudine non si guarisce più. È una malattia dolorosa che s'incunea nell'anima di alcuni, ma come un tarlo obbliga i poeti e i loro lettori (soprattutto gli scrittori) a pensare, a scrivere, a non darsi mai per vinti. $\grave{E}$ stata un morbo novecentesco, come ha scritto Tabucchi, ma può diventare la medicina di questi anni Duemila, che si presentano agli occhi di chi li vive come profondamente malati. 